\title{
Impaired oxygen uptake kinetics in heart failure with preserved ejection fraction
}

\author{
Christopher M Hearon Jr, ${ }^{\oplus 1,2}$ Satyam Sarma, ${ }^{1,2}$ Katrin A Dias, ${ }^{1,2}$ Michinari Hieda, ${ }^{1,2}$ \\ Benjamin D Levine ${ }^{1,2}$
}

- Additional material is published online only. To view please visit the journal online (http://dx.doi.org/10.1136/ heartjnl-2019-314797)

Department of Internal Medicine, University of Texas Southwestern Medical Center, Dallas, Texas, USA

${ }^{2}$ Texas Health Presbyterian Hospital Dallas, Institute for Exercise and Environmental Medicine, Dallas, Texas, USA

\section{Correspondence to}

Dr. Benjamin D Levine; BenjaminLevine@TexasHealth. org

Received 30 January 2019 Revised 10 May 2019 Accepted 14 May 2019 Published Online First 17 June 2019

\section{Linked}

- http://dx.doi.org/10.1136/ heartjnl-2019-315277

\section{Check for updates}

(C) Author(s) (or their employer(s)) 2019. No commercial re-use. See rights and permissions. Published by BMJ.

To cite: Hearon Jr CM Sarma S, Dias KA, et al. Heart 2019;105:1552-1558.

\begin{abstract}
Objective The time needed to increase oxygen utilisation to meet metabolic demand ( $\mathrm{VO}_{2}$ kinetics) is impaired in heart failure (HF) with reduced ejection fraction and is an independent risk factor for HF mortality. It is not known if $\mathrm{VO}_{2}$ kinetics are slowed in $\mathrm{HF}$ with preserved ejection fraction (HFpEF). We tested the hypothesis that $\mathrm{VO}_{2}$ kinetics are slowed during submaximal exercise in HFpEF and that slower $\mathrm{VO}_{2}$ kinetics are related to impaired peripheral oxygen extraction.
\end{abstract}

Methods Eighteen HFpEF patients (68 7 years, 10 women) and 18 healthy controls (69 66 years, 10 women) completed submaximal and peak exercise testing. Cardiac output (acetylene rebreathing, Q*), ventilatory oxygen uptake ( $\mathrm{VO}_{2}$, Douglas bags) and arterial-venous $\mathrm{O}_{2}$ difference ( $\mathrm{a}-\mathrm{v} \mathrm{O}_{2}$ difference) derived from $\mathrm{Q}_{c}$ and $\mathrm{VO}_{2}$ were assessed during exercise. Breath-by-breath $\mathrm{O}_{2}$ uptake was measured continuously throughout submaximal exercise, and $\mathrm{VO}_{2}$ kinetics was quantified as mean response time (MRT).

Results HFpEF patients had markedly slowed $\mathrm{VO}_{2}$ kinetics during submaximal exercise (MRT: control: 40.1 \pm 14.2, HFpEF: 65.4 \pm 27.7 s; $p<0.002$ ), despite no relative impairment in submaximal cardiac output (Q: control: 8.6 \pm 1.7, HFpEF: 9.7 $\pm 2.2 \mathrm{~L} / \mathrm{min} ; \mathrm{p}=0.79$ ). When stratified by MRT, HFpEF with an MRT $\geq 60 \mathrm{~s}$ demonstrated elevated $\mathrm{Q}_{\mathrm{C}^{\prime}}$, and impaired peripheral oxygen extraction that was apparent during submaximal exercise compared with HFpEF with a MRT $<60 \mathrm{~s}$ (submaximal a- $\mathrm{vO}_{2}$ difference: MRT $<60 \mathrm{~s}: 9.7 \pm 2.1$, MRT $\geq 60$ s: $7.9 \pm 1.1 \mathrm{~mL} / 100 \mathrm{~mL} ; p=0.03$ ).

Conclusion HFpEF patients have slowed $\mathrm{VO}_{2}$ kinetics that are related to impaired peripheral oxygen utilisation. MRT can identify HFpEF patients with peripheral

limitations to submaximal exercise capacity and may be a target for therapeutic intervention.

\section{INTRODUCTION}

$\mathrm{VO}_{2}$ kinetics reflect the speed at which oxygen delivery and utilisation rise to meet the metabolic demand of contracting skeletal muscle during the transition from rest to steady state exercise, which may be quantified as the mean response time (MRT). ${ }^{1}$ Impaired $\mathrm{VO}_{2}$ kinetics are a common characteristic of heart failure with reduced ejection fraction (HFrEF) that is closely associated with the severity of cardiac dysfunction. ${ }^{2-5}$ Impairments along every step of the oxygen transport and utilisation cascade have been identified in HFrEF, each contributing mechanistically to impaired
$\mathrm{VO}_{2}$ kinetics and exercise intolerance. However, a distinguishing feature of HFrEF is reduced cardiac output $\left(\mathrm{Q}_{\mathrm{c}}\right)$ at rest that remains depressed throughout submaximal and maximal exercise, such that oxygen uptake is partially limited by reduced bulk oxygen delivery to working skeletal muscle. ${ }^{67}$ HFrEF and HFpEF share many pathophysiological features that contribute to impaired $\mathrm{VO}$, kinetics including reduced skeletal muscle diffusive and oxidative capacity. ${ }^{8}$ However, a critical difference between these HF patients is the presence of an adequate $\mathrm{Q}_{c}$ response to submaximal exercise in heart failure with preserved ejection fraction (HFpEF) compared with HFrEF patients ${ }^{79}$ despite significant central limitations during maximal exercise in HFpEF. ${ }^{11}$

Similar to HFrEF, peak $\mathrm{Q}_{\mathrm{c}}$ and impaired peripheral oxidative capacity limit peak exercise performance in HFpEF patients. ${ }^{79-17}$ While peak $\mathrm{Q}_{c}$ is blunted due to chronotropic incompetence and impaired stroke volume reserve during maximal exercise $^{12}$ it is unclear to what extent $\mathrm{HFpEF}$ patients experience impaired haemodynamic and metabolic function during submaximal exercise, similar to activities of daily living. In contrast to HFrEF, HFpEF patients consistently demonstrate normal augmentation of $\mathrm{Q}$ during low-intensity submaximal exercise. ${ }^{791018}$ Thus, the pathophysiology of exercise intolerance during submaximal exercise may be distinct from HFrEF patients. However, to date, no studies have investigated how the unique pathophysiology observed in HFpEF impacts $\mathrm{VO}_{2}$ kinetics. Therefore, we tested the hypothesis that HFpEF patients would demonstrate slow $\mathrm{VO}_{2}$ kinetics (longer MRT) compared with age matched controls and that this abnormality may be primarily related to reduced peripheral oxidative capacity.

\section{METHODS}

\section{Subject characteristics}

A total of 18 patients with a hospital discharge diagnosis of CHF were enrolled in studies at the Institute for Exercise and Environmental Medicine between 2007 and 2016. Haemodynamic data from $11 \mathrm{HFpEF}$ patients in this investigation have been reported previously ${ }^{18}$ and are combined with data from seven subsequent HFpEF patients whose data have not been published previously. Detailed inclusion and exclusion criteria are provided in online supplementary materials. All subjects signed an informed consent form approved by the 
institutional review boards of UTSW Medical Center and Presbyterian Hospital, Dallas, Texas.

\section{Cardiopulmonary exercise testing (CPET)}

Subjects completed two modified Astrand-Saltin incremental treadmill protocols to determine peak oxygen uptake $\left(\mathrm{VO}_{2}\right.$ peak) as reported previously. ${ }^{18}$ On the first occasion, maximal exercise testing was used for screening and familiarisation (day 1). At least 72 hours later, exercise testing was repeated to assess steadystate haemodynamics, $\mathrm{VO}_{2}$ and $\mathrm{VO}_{2}$ kinetics during submaximal exercise, followed by haemodynamics and $\mathrm{VO}_{2}$ during maximal exercise (day 2). Resting haemodynamics and ventilatory $\mathrm{VO}_{2}$ were measured during $3 \mathrm{~min}$ of quiet standing on a treadmill. Following resting measurements, a low-intensity steady-state protocol was initiated at the desired treadmill velocity, enabling a rapid transition into the steady state workload. The workload was chosen for each individual to be below ventilatory threshold ( $\sim 40 \%-50 \%$ predicted $\mathrm{VO}_{2}$ peak, calculated from day 1 CPET) for $6 \mathrm{~min}$. After a minimum of $10 \mathrm{~min}$ of rest, peak exercise was assessed a second time using the same modified Astrand-Saltin incremental treadmill protocol as performed on day 1. Atrioventricular nodal blockers were withheld for 48 hours prior to avoid confounding effects of $\beta$-blockade on oxygen uptake kinetics (see online supplementary material S1). Other antihypertensive medications were continued.

Heart rate (HR) was monitored continuously via electrocardiogram (Schiller AT-10; Welch Allyn Inc, Skaneateles Falls, New York, USA), and blood pressure was measured using electrosphygmomanometry (Suntech Tango+; Morrisville, North Carolina, USA). Measurements of ventilatory gas exchange were made on a breath-by-breath basis using an infrared turbine flow metre (VMM, Interface Associates; Laguna Niguel, California, USA) and mass spectrometry for rapid gas analysis via proprietary software. In addition, breath-by-breath ventilatory gas exchange was also verified at steady state by collection of expired gas in a Douglas bag for $1 \mathrm{~min}$ and analysed via mass spectrometry. Steady-state ventilatory volume was measured by use of a 120 L Tissot spirometer (W.E. Collins P-1700; Braintree, Massachusetts, USA). During maximal exercise, peak $\mathrm{VO}_{2}$ was defined as the highest oxygen uptake measured from at least a 30 s Douglas bag collection. $\mathrm{Q}_{\mathrm{c}}$ was measured using a modified acetylene rebreathing technique that is well validated, reproducible and equivalent to invasive measures of $\mathrm{Q}_{c}$ during maximal exercise. ${ }^{18}$ Arterial-venous oxygen difference (a- $\mathrm{vO}_{2}$ difference) was calculated from the ratio between $\mathrm{Q}_{\mathrm{c}}$ and oxygen uptake using the Fick equation.

\section{$\mathrm{VO}_{2}$ kinetics}

$\mathrm{VO}_{2}$ kinetics were measured during the transition from rest to steady-state exercise ( $40 \%-50 \% \mathrm{VO}_{2}$ peak) as described above. Ventilatory $\mathrm{VO}_{2}$ data were linearly interpolated to $1 \mathrm{~s}$ intervals, time averaged into $5 \mathrm{~s}$ bins and fit using first-order kinetics (see online supplementary materials S2 and S3), with the time delay set to the start of exercise and therefore inclusive of both the cardiodynamic phase I and phase II rise in $\mathrm{VO}_{2}$. MRT was defined as the exponential time constant $\tau$, which represents the time needed for oxygen uptake to rise to $\sim 63 \%$ of steady state $\mathrm{VO}_{2}$. Oxygen deficit was calculated using Simpson's method as the difference between cumulative oxygen demand and cumulative oxygen utilisation.

\section{Statistical analysis}

The sample was selected from all of the CPET tests collected as part of research studies at the Institute for Exercise and
Environmental medicine between 2007 and 2016 (18 and online supplementary materials S5 and S6) to maximise sample size. Inclusion/exclusion of all tests was determined prior to statistical analysis, and the sample represents all tests with adequate breath by breath and haemodynamic data available at IEEM for this particular analysis. The typical error of $\mathrm{VO} 2$ uptake kinetics (specifically MRT) in our laboratory is $8 \%-13 \%$ (or 5-8 s; see online supplementary material S7). Various physiological variables are reported as descriptive variables of the experimental intervention in both groups. Primary comparisons of MRT and a-vO difference between control and HFpEF patients was made using unpaired Student's t-tests. As an exploratory post hoc analysis, HFpEF patients were stratified into fast and slow kinetics based on an MRT of $60 \mathrm{~s}$ (MRT $<60 \mathrm{~s}$ and MRT $\geq 60$ s, respectively). Comparison of haemodynamics were made separately during rest, the $\mathrm{VO}_{2}$ kinetics protocol and the graded exercise testing protocol between control, MRT $<60 \mathrm{~s}$ and MRT $\geq 60 \mathrm{~s}$ using analysis of variance and Newman-Keuls post hoc tests, which is robust up to three comparison groups but does not adjust for multiple comparisons. Fishers' exact test was used for categorical data. Significance was set a priori at $\mathrm{p}<0.05$. Data for all primary comparisons are expressed in text as mean difference with $95 \%$ CIs, and all data were expressed in tabular form as mean $\pm \mathrm{SD}$.

\section{RESULTS}

\section{Subject characteristics}

Clinical characteristics of control subjects $(n=18)$, HFpEF patients $(n=18)$ and HFpEF patients stratified by MRT (MRT <60 s: $n=9$; MRT $\geq 60$ s: $n=9$ ) are presented in table 1 .

\section{$\mathrm{VO}_{2}$ kinetics and haemodynamics during submaximal exercise: HFpEF versus control}

During submaximal exercise, there was no evidence of chronotropic incompetence, and the rise in $\mathrm{Q}_{c}$ was not different between HFpEF and controls ( $\triangle \mathrm{Qc}$ : control: $4.61 \pm 1.02 \mathrm{vs}$ HFpEF: $4.82 \pm 1.62 \mathrm{~L} / \mathrm{min} ; \mathrm{p}=0.65)$. Submaximal a-vO2 difference $(\mathrm{mL} / 100 \mathrm{~mL})$ was not different between HFpEF and controls (mean difference: $0.96 \mathrm{~mL} / 100 \mathrm{~mL}, 95 \%$ CI -0.286 to $2.204 ; \mathrm{p}=0.18)$. There were no significant differences in oxygen demand $\left(\mathrm{VO}_{2}\right)$ or the amplitude of $\mathrm{VO}_{2}$ kinetics

\begin{tabular}{|c|c|c|c|c|}
\hline Characteristic & $\begin{array}{l}\text { Control } \\
(n=18)\end{array}$ & $\begin{array}{l}\text { HFpEF } \\
(n=18)\end{array}$ & $\begin{array}{l}\text { HF MRT }<60 \text { s } \\
(n=9)\end{array}$ & $\begin{array}{l}\text { HF MRT } \geq 60 S \\
(n=9)\end{array}$ \\
\hline Sex (M:F) & $8: 10$ & $8: 10$ & 4:5 & $4: 5$ \\
\hline Age (year) & $70(7)$ & $68(7)$ & $68(7)$ & $69(7)$ \\
\hline Height (cm) & $167(10)$ & $167(9)$ & 167 (8) & $166(10)$ \\
\hline Weight (kg) & 75 (12) & $99(19)^{*}$ & $100(15)^{*}$ & $98(24)^{*}$ \\
\hline BMI $\left(\mathrm{kg} / \mathrm{m}^{2}\right)$ & $26(3)$ & $35(6)^{*}$ & $36(4)^{*}$ & $35(7)^{*}$ \\
\hline $\mathrm{BSA}\left(\mathrm{m}^{2}\right)$ & $1.85(0.19)$ & $2.13(0.26)^{*}$ & $2.15(0.2)^{*}$ & $2.11(0.3)^{*}$ \\
\hline \multicolumn{5}{|c|}{ HF pharmacotherapy, n (\%) } \\
\hline Diuretic & & $16(89)$ & $7(78)$ & $9(100)$ \\
\hline ACEi or ARB & & $11(61)$ & $4(44)$ & $7(78)$ \\
\hline$\beta$-blocker & & $11(61)$ & $4(44)$ & $7(78)$ \\
\hline Nitrate & & $4(22)$ & $1(11)$ & $3(33)$ \\
\hline Statin & & $8(44)$ & $3(33)$ & $5(56)$ \\
\hline Aspirin & & $6(33)$ & 1 (11) & $5(56)$ \\
\hline
\end{tabular}


A

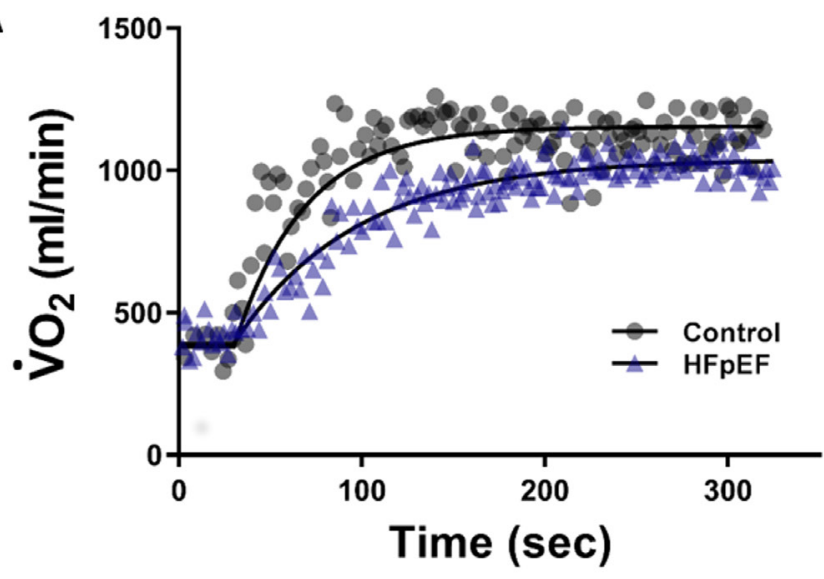

B

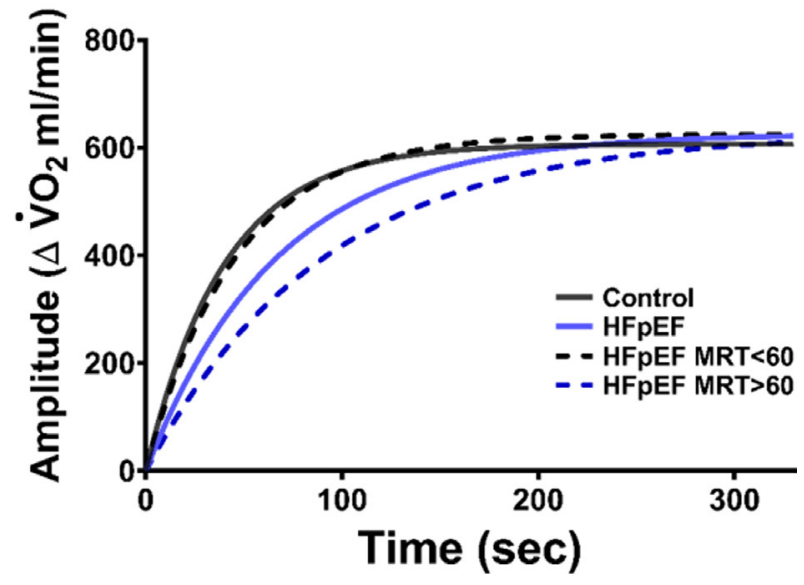

Figure 1 (A) Representative breath-by-breath ventilatory oxygen uptake kinetics in a HFpEF patient (blue) and a healthy control subject (black). Breath-by-breath oxygen utilisation $\left(\mathrm{VO}_{2}\right)$ was measured during submaximal treadmill exercise, interpolated linearly at $1 \mathrm{~s}$ intervals and fitted using on-transient monoexponential curve fitting. (B) Mean response curves for controls HFpEF patients and HFpEF patients stratified by mean response time (MRT) modelled using group average amplitude $\left(\Delta \mathrm{VO}_{2}=\right.$ steady state $\mathrm{VO}_{2}-$ baseline $\mathrm{VO}_{2}$ ) and $\mathrm{MRT}$, defined as the exponential time constant of $\mathrm{VO}_{2}$ onset kinetics, which reflects the time needed for oxygen uptake to rise to $63 \%$ of steady state $\mathrm{VO}_{2}$. HFpEF, heart failure with preserved ejection fraction.

$\left(\Delta \mathrm{VO}_{2}\right)$, defined as the rise in $\mathrm{VO}_{2}$ from baseline to steady state (control: $0.61 \pm 0.15$ vs HFpEF: $0.63 \pm 0.15 \mathrm{~L} / \mathrm{min} ; \mathrm{p}=0.70$ ). However, MRT was much longer in HFpEF patients (mean difference: -25.3 s, $95 \%$ CI 10.4 to $40.2 ; \mathrm{p}=0.002$ ) nearly doubling the oxygen deficit (control: $0.38 \pm 0.23$ vs HFpEF: $0.63 \pm 0.28 \mathrm{~L}$; $\mathrm{p}=0.007$ ) (figures 1 and 2).

Haemodynamics at peak exercise: HFpEF versus control At peak exercise, absolute $\mathrm{VO}_{2}$ was slightly but variably lower in HFpEF compared with controls (control: $1.65 \pm 0.44 \mathrm{vs}$ HFpEF: $1.44 \pm 0.48 \mathrm{~L} / \mathrm{min} ; \mathrm{p}=0.19$ ). Peak $\mathrm{VO}_{2}$ was consistently and significantly lower in HFpEF patients when scaled to body weight (control: $22.2 \pm 4.0$ vs HFpEF: $14.6 \pm 3.1 \mathrm{~mL}$ / $\mathrm{min} / \mathrm{kg} ; \mathrm{p}<0.001$ ). There was no difference in peak Qic between groups (control: $13.4 \pm 4.0$ vs HFpEF: $13.9 \pm 3.5 \mathrm{~L} / \mathrm{min} ; \mathrm{p}=0.69$ ) (table 4). In contrast to submaximal exercise, significant impairments in peripheral a- $\mathrm{vO}_{2}$ difference were apparent at peak exercise in HFpEF patients (mean difference: $-2.41 \mathrm{~mL} / 100 \mathrm{~mL}$,
A

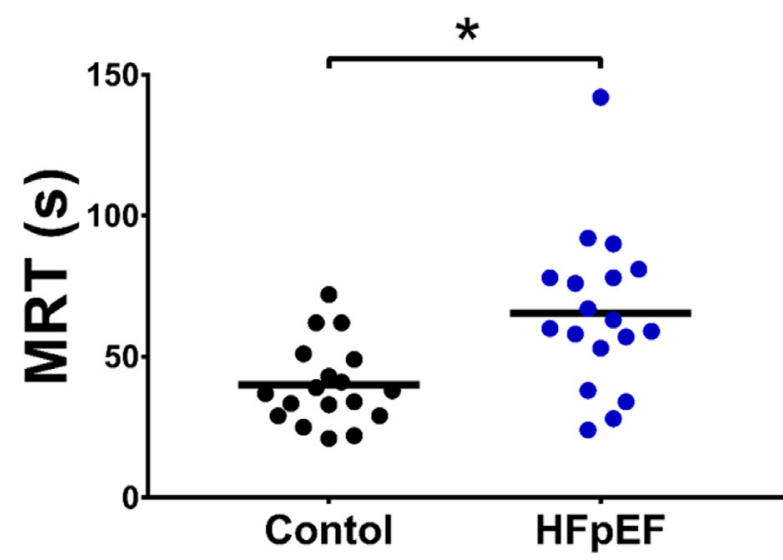

B

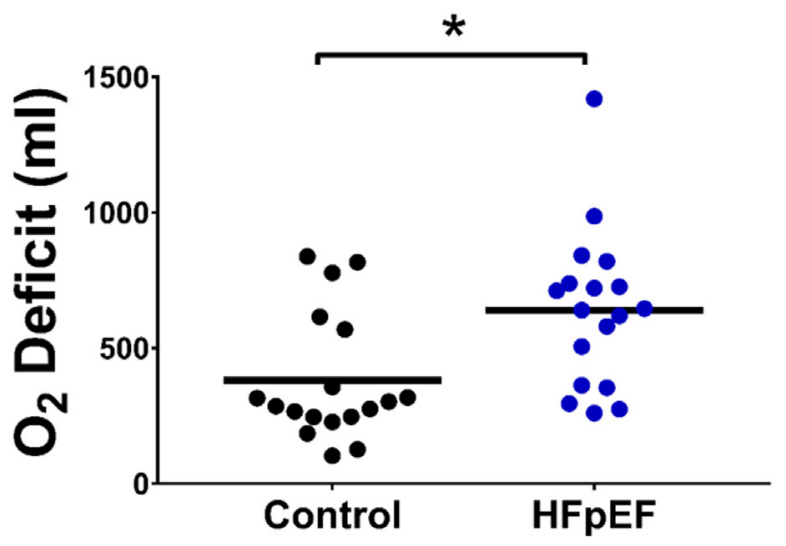

Figure 2 Mean response time (MRT) is slower and oxygen deficit is greater in HFpEF patients. The MRT in seconds (s) of oxygen uptake kinetics $(A)$ and accumulated $\mathrm{O}_{2}$ deficit $(\mathrm{B})$ during submaximal treadmill exercise in HFpEF patients and age-matched healthy controls. The MRT of oxygen uptake kinetics were slower, and $\mathrm{O}_{2}$ deficit was exaggerated in $\mathrm{HFpEF}$ patients compared with controls. ${ }^{*} \mathrm{P}<0.01$ versus controls; $\mathrm{n}=18,8 \mathrm{M}: 10 \mathrm{~F}$ for each group, respectively. $\mathrm{HFpEF}$, heart failure with preserved ejection fraction.

$95 \%$ CI -0.59 to $-4.231 ; \mathrm{p}=0.01$ ) consistent with previous studies performed in the upright posture.7 101219 Peak heart rate was lower in HFpEF patients compared with controls (control: $158 \pm 13$ vs HFpEF: $132 \pm 23 \mathrm{bpm}$; $\mathrm{p}=0.01$ ), and there were no significant differences between groups in blood pressure (systolic, diastolic and mean arterial pressure), peak cardiac index or peak stroke volume index.

\section{Haemodynamics in HFpEF patients with MRT $<60$ s and} MRT $\geq 60$ s

To better understand the impact of slowed kinetics on exercise performance, HFpEF patients were stratified using an MRT of $60 \mathrm{~s}^{2}{ }^{4}$ creating two subgroups: HFpEF with normal MRT (MRT <60s) and HFpEF with slowed MRT (MRT $\geq 60 \mathrm{~s}$ ). Previous investigations have demonstrated that MRTs $\geq 60 \mathrm{~s}$ in HFrEF patients are predictive of reduced survival, transplant free survival and survival free of hospitalisation. ${ }^{4-6}$ Subject characteristics, haemodynamics at rest, submaximal and peak exercise are presented in tables 1-4. During submaximal exercise, there was no difference in baseline $\mathrm{VO}_{2}$, steady state $\mathrm{VO}_{2}$ and $\mathrm{VO}_{2}$ amplitude between the HFpEF cohorts (table 3). In HFpEF patients with MRT $\geq 60 \mathrm{~s}$, peripheral oxygen extraction 
Table 2 Resting haemodynamics in healthy controls and patients with HFpEF

\begin{tabular}{|c|c|c|c|c|}
\hline Variable & $\begin{array}{l}\text { Control } \\
(n=18)\end{array}$ & $\begin{array}{l}\text { HFpEF } \\
(n=18)\end{array}$ & $\begin{array}{l}\text { HF MRT }<60 s \\
(n=9)\end{array}$ & $\begin{array}{l}\text { HF MRT } \geq 60 s \\
(n=9)\end{array}$ \\
\hline $\mathrm{SBP}(\mathrm{mm} \mathrm{Hg})$ & $132(21)$ & $130(24)$ & $120(17)$ & $133(15)$ \\
\hline $\mathrm{DBP}(\mathrm{mm} \mathrm{Hg})$ & $83(8)$ & $74(12)^{*}$ & $73(12)$ & $72(11)$ \\
\hline MAP $(\mathrm{mm} \mathrm{Hg})$ & 100 (11) & $93(14)$ & $89(11)$ & $92(10)$ \\
\hline HR (beats/min) & $76(12)$ & $79(17)$ & 80 (19) & $77(16)$ \\
\hline$Q_{c}(L / m i n)$ & $4.0(1.0)$ & $4.9(1.4)^{*}$ & $4.2(1.0)$ & $5.5(1.5)^{*} \dagger$ \\
\hline$Q_{c}$ index $\left(L / m i n / m^{2}\right)$ & $2.1(0.4)$ & $2.3(0.7)$ & $2.0(0.5)$ & $2.6(0.7)^{*} \dagger$ \\
\hline $\mathrm{SV}(\mathrm{mL})$ & $53(18)$ & $63(20)$ & $54(20)$ & $71(16)^{*}$ \\
\hline $\mathrm{SV}$ index $\left(\mathrm{mL} / \mathrm{m}^{2}\right)$ & $28(8)$ & $30(11)$ & $25(10)$ & $34(10)^{*}$ \\
\hline TPR (dyne $/ \mathrm{s} / \mathrm{cm}^{5}$ ) & $2106(508)$ & $1607(325)^{*}$ & $1736(334)$ & $1477(277)^{*}$ \\
\hline $\mathrm{VO}_{2}(\mathrm{~L} / \mathrm{min})$ & $0.22(0.05)$ & $0.30(0.11)^{*}$ & $0.28(0.1)$ & $0.32(0.1)^{*}$ \\
\hline $\mathrm{VO}_{2}(\mathrm{~mL} / \mathrm{kg} / \mathrm{min})$ & $2.9(0.5)$ & $3.2(1.1)$ & $2.8(0.5)$ & $3.6(1.3)$ \\
\hline $\begin{array}{l}\mathrm{a}-\mathrm{vO}_{2} \text { difference } \\
(\mathrm{mL} / 100 \mathrm{~mL})\end{array}$ & $5.7(1.7)$ & $6.4(2.1)$ & $7.02(2.4)$ & $5.74(1.8)$ \\
\hline \multicolumn{5}{|c|}{ 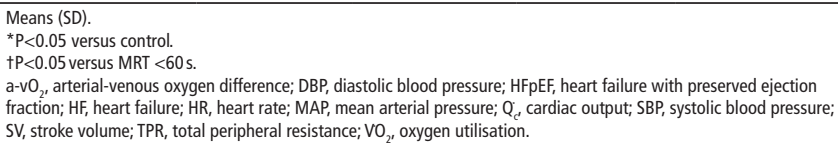 } \\
\hline
\end{tabular}

was reduced during submaximal exercise compared with control and MRT <60s (a-vO2 difference: control: 9.8 \pm 1.8 ; MRT $<60$ s: $9.7 \pm 2.1$; MRT $\geq 60$ s: $7.9 \pm 1.1 \mathrm{~mL} / 100 \mathrm{~mL}$; $\mathrm{p}<0.05)$. These patients also required a greater $\mathrm{Q}_{c}$ for the same absolute $\mathrm{VO}_{2}$ (Control: $8.6 \pm 1.7$; MRT <60 s: $8.7 \pm 2.0$; MRT $\geq 60$ s: $10.7 \pm 2.1 \mathrm{~L} / \mathrm{min} ; \mathrm{p}<0.05)$. With the exception of blood pressure (MAP: MRT <60 s: $105 \pm 14$ vs MRT $\geq 60$ s: $122 \pm 13 \mathrm{~mm} \mathrm{Hg}$ ), there were no other significant differences in haemodynamics at peak exercise between MRT <60s and MRT $\geq 60$ s.

\section{DISCUSSION}

This investigation found that patients with HFpEF have significantly slowed $\mathrm{VO}_{2}$ kinetics during submaximal exercise compared with age-matched sedentary controls. The impairment in $\mathrm{VO}_{2}$ kinetics is similar in magnitude to that reported in HFrEF despite preserved $\mathrm{Q}_{c}$ during submaximal exercise. When $\mathrm{HFpEF}$ patients were stratified by MRT, those with an MRT $\geq 60 \mathrm{~s}$ showed an impairment in peripheral oxidative capacity earlier during submaximal exercise, similar in intensity to activities of daily living, which may result in rapid accumulation of oxygen deficit. Together, these findings suggest that oxygen transport and utilisation is severely compromised during submaximal exercise in HFpEF patients that may contribute significantly to exercise intolerance and fatigue.

\section{$\mathrm{VO}_{2}$ kinetics in heart failure (HF)}

In $\mathrm{HF}$ and other clinical populations, slower kinetics extend the time required for oxygen delivery to meet metabolic demand resulting in a large 'oxygen deficit' and fatigue. ${ }^{20}$ In this cohort of HFpEF patients, MRT was markedly (>50\%) impaired compared with the MRT of age-matched controls resulting in greater than 50\% increase in oxygen deficit (figure 2). Interestingly, the magnitude of impairment in MRT in HFpEF is similar to MRT values reported previously for HFrEF patients. ${ }^{2} 4$ 21-23 The presence of impaired MRT despite preserved submaximal $\mathrm{Q}_{\text {, }}$, a common finding in HFpEF patients, ${ }^{9} 101418$ underscores extensive impairments in vascular and metabolic capacity in HFpEF patients. The physiological consequence of such severely slowed $\mathrm{VO}_{2}$ kinetics is fatigue related to the rapid depletion of intramuscular high-energy phosphates. Indeed, assessment of intramuscular energetic parameters using ${ }^{31} \mathrm{P}$ magnetic resonance spectroscopy during plantar flexion exercise in HFpEF and HFrEF patients revealed a greater rate of phosphocreatine decline in HFpEF patients, which correlate with skeletal muscle fatigue (plantar flexion), peak $\mathrm{VO}_{2}$ and $6 \mathrm{~min}$ walk performance. $^{24}$

Table $3 \mathrm{VO}_{2}$ kinetics and submaximal exercise haemodynamics in controls and HFpEF patients

\begin{tabular}{|c|c|c|c|c|}
\hline Variable & Control & HFpEF & HF MRT $<60 \mathrm{~s}$ & $\mathrm{HF} M \mathrm{MRT} \geq 60 \mathrm{~s}$ \\
\hline \multicolumn{5}{|l|}{$\mathrm{VO}_{2}$ kinetics } \\
\hline Amplitude (L/min) & $0.61(0.15)$ & $0.63(0.15)$ & $0.63(0.15)$ & $0.63(0.15)$ \\
\hline MRT (s) & $40.1(14.2)$ & $65.4(27.7)^{*}$ & $45.6(14.7)$ & $85.2(23.2)^{*} \dagger$ \\
\hline $\mathrm{O}_{2}$ deficit (L) & $0.38(0.23)$ & $0.63(0.28)^{*}$ & $0.47(0.20)$ & $0.80(0.28)^{*} \dagger$ \\
\hline \multicolumn{5}{|l|}{ Steady state } \\
\hline $\mathrm{SBP}(\mathrm{mm} \mathrm{Hg})$ & $153(27)$ & $156(28)$ & $145(28)$ & $158(24)$ \\
\hline $\mathrm{DBP}(\mathrm{mm} \mathrm{Hg})$ & $86(16)$ & $76(16)$ & $80(14)$ & $70(17)$ \\
\hline MAP (mm Hg) & $110(15)$ & $102(16)$ & $102(16)$ & $100(16)$ \\
\hline HR (beats/min) & $98(16)$ & $99(17)$ & $101(21)$ & $97(12)$ \\
\hline$Q_{c}(L / m i n)$ & $8.6(1.7)$ & $9.7(2.2)$ & $8.7(2.0)$ & $10.7(2.1)^{*} \dagger$ \\
\hline$Q_{c}$ index $\left(L / m i n / m^{2}\right)$ & $4.6(0.8)$ & $4.6(1.0)$ & $4.1(0.9)$ & $5.1(0.7) \dagger$ \\
\hline $\mathrm{SV}(\mathrm{mL})$ & 89 (28.4) & $99(20)$ & 87 (14) & $111(18)^{*} \dagger$ \\
\hline TPR (dyne/s/cm & $1048(253)$ & $876(192)^{*}$ & $962(179)^{*}$ & $791(163)^{*}$ \\
\hline SV index $\left(\mathrm{mL} / \mathrm{m}^{2}\right)$ & $48(13)$ & $47(10)$ & $41(6)$ & $53(10) \dagger$ \\
\hline $\mathrm{VO}_{2}(\mathrm{~L} / \mathrm{min})$ & $0.81(0.1)$ & $0.85(0.2)$ & $0.83(0.2)$ & $0.84(0.2)$ \\
\hline $\mathrm{VO}_{2}(\mathrm{~mL} / \mathrm{kg} / \mathrm{min})$ & $11.4(1.3)$ & $8.7(1.9)^{*}$ & $8.3(1.6)^{*}$ & $9.0(2.2)^{*}$ \\
\hline $\mathrm{VO}_{2}(\%$ peak $)$ & $52(12)$ & $61(12)^{*}$ & $59(9)^{*}$ & $61(14)^{*}$ \\
\hline $\mathrm{a}-\mathrm{vO}_{2}$ difference $(\mathrm{mL} / 100 \mathrm{~mL})$ & $9.8(1.8)$ & $8.9(1.9)$ & $9.7(2.1)$ & $7.9(1.1)^{*} \dagger$ \\
\hline$\Delta \mathrm{a}-\mathrm{vO}_{2}$ difference $(\mathrm{mL} / 100 \mathrm{~mL})$ & $4.1(1.1)$ & $2.5(1.7)^{*}$ & $2.7(1.9)^{*}$ & $2.2(1.5)^{*}$ \\
\hline
\end{tabular}

Means (SD).

${ }^{*} \mathrm{P}<0.05$ versus control.

$\dagger \mathrm{P}<0.05$ versus $M R T<60 \mathrm{~s}$.

a- $\mathrm{VO}_{2}$, arterial-venous oxygen difference; $\mathrm{DBP}$, diastolic blood pressure; $\mathrm{HFpEF}$, heart failure with preserved ejection fraction; $\mathrm{HF}$, heart failure; $\mathrm{HR}$, heart rate; MAP, mean arterial pressure; $Q_{2}$, cardiac output; $S B P$, systolic blood pressure; $S V$, stroke volume; TPR, total peripheral resistance; $\mathrm{VO}_{2}$, oxygen utilisation. 
To further explore the relationship between MRT and submaximal exercise haemodynamics, we stratified HFpEF patients into two groups based on an MRT cut-off of $60 \mathrm{~s}$. Previous studies investigating the predictive value of MRT indicate that HFrEF patients with an MRT $\geq 60$ s have lower rates of survival, transplant free survival and survival free of hospitalisation compared with HFrEF patients with an MRT <60 s. ${ }^{2} 325$ Additionally, HFrEF patients with an MRT $\geq 60$ s have significantly worse indices of cardiac function including lower ejection fraction and elevated pulmonary arterial pressure during exercise. ${ }^{4} 2126$ In this investigation, HFpEF patients with normal MRT experienced similar reductions in peak exercise capacity as patients with $\mathrm{MRT} \geq 60$ s (peak $\mathrm{VO}_{2}$ : control: $22.2 \pm 4$, MRT $<60$ s: $14.6 \pm 3.1$, MRT $\geq 60 \mathrm{~s}: 14.7 \pm 2.5 \mathrm{~mL} / \mathrm{kg} / \mathrm{min}$; both $\mathrm{p}<0.05$ vs control), demonstrating that slowed MRT is not merely a generalised consequence of deconditioning.

Rather, slow MRT identified a unique phenotype within HFpEF patients characterised by disproportionately impaired peripheral oxygen extraction. Clinically, patients from each 'subtype' of HFpEF (slower vs faster MRT) would have appeared to be identical based solely on peak $\mathrm{VO}_{2}$ measured during standard CPET. However, incorporation of $\mathrm{VO}_{2}$ kinetics was able to further distinguish between these HFpEF phenotypes. The 'peripherally limited' HFpEF phenotype with impaired MRT may be particularly susceptible to exercise intolerance and fatigue given the presence of impaired oxidative capacity during submaximal exercise intensities compared with HFpEF with normal MRT. Assessment of MRT may identify those patients that are well suited for training interventions targeted at improving skeletal muscle function.

\section{Potential mechanisms for reduced kinetics in HFpEF}

In healthy individuals, $\mathrm{VO}_{2}$ kinetics are largely independent of oxygen delivery and instead constrained by mitochondrial capacity. ${ }^{1}$ However, in the case of pathophysiological limitations of $\mathrm{Q}_{c}$, like that observed in $\mathrm{HFrEF}, \mathrm{VO}_{2}$ kinetics may become oxygen delivery dependent. Recently, Chatterjee $e t ~ a l^{4}$ assessed both central and peripheral determinants of oxygen uptake concurrently with MRT to identify the factors related to slow MRT in patients with HFrEF. They report that slowed kinetics (MRT $\geq 60 \mathrm{~s}$ ) are associated with reduced $\mathrm{Q}_{\mathrm{c}}$ and exaggerated peripheral oxygen extraction during submaximal and peak exercise. The presence of reduced $\mathrm{Q}_{\mathrm{c}}$ and exaggerated peripheral extraction is consistent with a significant limitation of $\mathrm{Q}_{c}$ and bulk oxygen delivery to contracting skeletal muscle, which is partially compensated by elevated peripheral oxygen extraction in HFrEF patients. ${ }^{27}$ In contrast to HFrEF, impaired MRT in HFpEF was present despite a normal or exaggerated $Q_{c}$ response to submaximal exercise and was associated with an attenuated rise in a-vO $\mathrm{v}_{2}$ difference $\left(\Delta \mathrm{a}-\mathrm{vO}_{2}\right.$ diff: control: $4.1 \pm 1.1$, HFpEF: $2.5 \pm 1.7 \mathrm{~mL} / 100 \mathrm{~mL} ; \mathrm{p}=0.002)$ suggestive of a primary contribution of impaired skeletal muscle oxidative capacity.

Importantly, the finding of preserved submaximal $\mathrm{Q}_{\mathrm{c}}$ and reduced $\mathrm{a}-\mathrm{vO}_{2}$ difference during exercise is not isolated to this cohort of HFpEF patients. A recent meta-analysis indicates that HFpEF patients have normal or slightly smaller changes in $\mathrm{a}-\mathrm{vO}_{2}$ difference at peak exercise in the supine position, whereas during exercise in the upright posture (the position of most activities of daily living), a-vO difference at peak exercise is consistently lower. ${ }^{12}$ In regards to $\mathrm{Q}_{\mathrm{c}}$, all studies to date have found that HFpEF patients display a preserved or exaggerated $\mathrm{Q}_{\mathrm{c}}$ response during submaximal exercise. ${ }^{9} 101418$ Specifically, the majority of these studies indicate that the rise in $\mathrm{Q}_{c}$ in response to a change in $\mathrm{VO}_{2}\left(\mathrm{Q} / \mathrm{VO}_{2}\right.$ relationship) is preserved in $\mathrm{HFpEF}$ patients compared with healthy controls. ${ }^{10} 15-182829$ These findings indicate that at sufficiently mild exercise intensities when $\mathrm{Q}_{\mathrm{c}}$ is not limited, skeletal muscle and vascular maladaptations may be important for understanding the impairment in $\mathrm{VO}_{2}$ kinetics and exercise intolerance in HFpEF.

Furthermore, Kitzman and colleagues ${ }^{8}$ show reduced capillaryto-fibre ratio, and a shift towards glycolytic type II muscle fibres in skeletal muscle of HFpEF patients. Greater percentage of type II fibres has been tied mechanistically to slower $\mathrm{VO}_{2}$ kinetics in healthy individuals (see online supplementary material). Several studies also report a smaller change in $\mathrm{VO}_{2}$ in response to a given change in external work rate in HFpEF, ${ }^{10} 1428$ consistent with impaired oxidative capacity and greater prevalence of type 2 fibres. These skeletal muscle fibre type, and structural abnormalities may be compounded further by dysregulated mitochondrial function related to obesity and excessive skeletal muscle fat deposition ${ }^{2430}$ that result in cellular metabolic impairment, rapid depletion of high energy phosphates and muscular fatigue. ${ }^{24}$ Emerging evidence suggests that these pathophysiological skeletal muscle abnormalities may be primary features of an obese HFpEF phenotype. ${ }^{15}$ In some HFpEF patients, skeletal muscle abnormalities are present during submaximal exercise intensities and manifest prominently during metabolic transitions. ${ }^{724} 30$ Future studies using more direct measures of skeletal muscle oxidative function will be important to determine the mechanisms and extent of skeletal muscle impairments in HFpEF patients. Significant limitations may also exist upstream of skeletal muscle in the oxygen cascade including ventilatory gas exchange, vascular convective oxygen delivery, the distribution of blood flow to, and within active skeletal muscle, and diffusive capacity of oxygen from blood to skeletal muscle.

\section{Limitations and considerations}

HFpEF patients demonstrate reduced chronotropy at peak exercise (table 4), ${ }^{12}$ and it is possible that slower HR kinetics during the transition from rest to steady state could contribute to lower $\mathrm{Q}_{\mathrm{c}}$ and thus slower $\mathrm{VO}_{2}$ kinetics. This study is not able to exclude a possible contribution of impaired $\mathrm{Q}_{\mathrm{c}}$ to slowed uptake kinetics. However, it is unlikely that chronotropic incompetence can explain the observed decrements in $\mathrm{VO}_{2}$ kinetics considering there was no evidence of lower HR during submaximal exercise in this study, and even at peak exercise, the lower HR did not reduce peak $Q_{c}$. However, further studies are warranted to fully characterise the relative contribution of on-transient cardiac responses to impairments in MRT in HFpEF patients. Finally, larger prospective studies will be necessary to validate the phenotypic findings of the MRT > 60 ssubanalysis in this investigation.

\section{CONCLUSION}

The MRT of $\mathrm{VO}_{2}$ kinetics, an independent predictor of hospitalisation and mortality in $\mathrm{HF}$, is significantly prolonged in HFpEF patients and may be related to impairments in peripheral oxidative capacity. Furthermore, stratification by MRT identified a subset of HFpEF patients with particularly severe peripheral oxidative limitations to submaximal exercise capacity. Given the complex multifactorial nature of $\mathrm{HFpEF}$, the primary mechanism of exercise intolerance is likely to be heterogeneous among patients. In contrast to a- $\mathrm{vO}_{2}$ difference, MRT can be assessed without measurement of $\mathrm{Q}_{\mathrm{c}}$ and may be useful clinically in conjunction with CPET to determine the 'physiological phenotype' of an individual HFpEF patient and 
Table 4 Haemodynamics at peak exercise in controls and HFpEF patients

\begin{tabular}{|c|c|c|c|c|}
\hline Variable & Control & HFpEF & HF MRT $<60 \mathrm{~s}$ & $\mathrm{HF} M \mathrm{MRT} \geq 60 \mathrm{~s}$ \\
\hline $\mathrm{SBP}(\mathrm{mm} \mathrm{Hg})$ & $192(24)$ & $178(36)$ & $159(34)^{*}$ & $188(28) \dagger$ \\
\hline $\mathrm{DBP}(\mathrm{mm} \mathrm{Hg})$ & $88(16)$ & $83(12)$ & $77(12)$ & $88(11)$ \\
\hline $\operatorname{MAP}(\mathrm{mm} \mathrm{Hg})$ & $122(13)$ & $114(16)$ & $105(14)^{*}$ & $122(13) \dagger$ \\
\hline $\mathrm{HR}$ (beats/min) & $158(13)$ & $132(23)^{*}$ & $129(28)^{*}$ & $134(19)^{*}$ \\
\hline$Q_{c}(L / m i n)$ & $13.4(4.0)$ & $13.9(3.5)$ & $13.3(3.8)$ & $14.5(3.2)$ \\
\hline $\mathrm{Q}_{c}$ index $\left(\mathrm{L} / \mathrm{min} / \mathrm{m}^{2}\right)$ & $7.2(1.8)$ & $6.6(1.47)$ & $6.2(1.7)$ & $6.9(1.2)$ \\
\hline $\mathrm{SV}(\mathrm{mL})$ & $85(25)$ & $104(15)^{*}$ & $100(2)$ & $108(17)^{*}$ \\
\hline SV index $\left(\mathrm{mL} / \mathrm{m}^{2}\right)$ & $45(12)$ & $49(8)$ & $47(6)$ & $52(10)$ \\
\hline TPR (dyne $/ \mathrm{s} / \mathrm{cm}^{5}$ ) & $801(287)$ & 695 (151) & $679(141)$ & 709 (167) \\
\hline $\mathrm{VO}_{2}(\mathrm{~L} / \mathrm{min})$ & $1.65(0.44)$ & $1.44(0.48)$ & $1.46(0.5)$ & $1.42(0.5)$ \\
\hline $\mathrm{VO}_{2}(\mathrm{~mL} / \mathrm{kg} / \mathrm{min})$ & $22.2(4.0)$ & $14.6(3.1)^{*}$ & $14.6(3.8)^{*}$ & $14.7(2.5)^{*}$ \\
\hline $\mathrm{a}-\mathrm{vO}_{2}$ difference $(\mathrm{mL} / 100 \mathrm{~mL})$ & $12.7(2.9)$ & $10.4(2.2)^{*}$ & $11.1(2.5)$ & $9.71(1.7)^{*}$ \\
\hline$\Delta \mathrm{a}-\mathrm{vO}_{2}$ difference $(\mathrm{mL} / 100 \mathrm{~mL})$ & $7.0(1.6)$ & $4.1(2.3)^{*}$ & $4.3(2.4)^{*}$ & $4.0(2.4)^{*}$ \\
\hline
\end{tabular}

Means (SD).

${ }^{*} \mathrm{P}<0.05$ versus control.

$\mathrm{t} P<0.05$ versus $M R T<60 \mathrm{~s}$.

$\mathrm{a}-\mathrm{vO}_{2}$, arterial-venous oxygen difference; $\triangle \mathrm{AvO}_{2}$ difference, change from baseline; $\mathrm{DBP}$, diastolic blood pressure; $\mathrm{HFpEF}$, heart failure with preserved ejection fraction; $\mathrm{HR}$, heart rate; MAP, mean arterial pressure; MRT, mean response time; $Q_{c^{\prime}}$ cardiac output; $S B P$, systolic blood pressure; $S V$, stroke volume; TPR, total peripheral resistance; $V_{2}$, oxygen utilisation.

identify patients who may benefit from exercise interventions tailored to improve skeletal muscle function. Finally, utilising MRT as a gauge of intervention efficacy may identify strategies that improve the integrative cardiovascular response to submaximal exercise intensities that are in line with activities of daily living, which may be missed when focusing solely on changes in peak $\mathrm{VO}_{2}$.

\section{Key messages}

\section{What is already known on this subject?}

$-\mathrm{O}_{2}$ kinetics are an integrative marker of the efficiency of the cardiovascular system and skeletal muscle to adapt to changes in metabolic demand. Addition of $\mathrm{VO}_{2}$ kinetics analysis to maximal exercise testing results improves prognostic capacity in heart failure with reduced ejection fraction.

\section{What might this study add?}

- In heart failure with preserved ejection fraction (HFpEF), $\mathrm{VO}_{2}$ kinetics are considerably slowed despite preserved cardiac output during submaximal exercise and are related to impairments in peripheral oxygen utilisation.

\section{How might this impact on clinical practice?}

- $\mathrm{O}_{2}$ kinetics assessed during submaximal exercise intensities can be incorporated into standard cardiopulmonary exercise testing to provide a more integrative assessment of cardiovascular and skeletal muscle function in addition to peak oxygen uptake. In $\mathrm{HFpEF}$, $\mathrm{VO}_{2}$ kinetics may be used to phenotype patients with significant peripheral limitations to exercise capacity and inform decisions regarding treatment and exercise rehabilitation.

Contributors All authors provided substantial contributions to the conception or design of the work or the acquisition, analysis or interpretation of data for the work; drafting the work or revising it critically for important intellectual content; final approval of the version to be published; and agreement to be accountable for all aspects of the work in ensuring that questions related to the accuracy or integrity of any part of the work are appropriately investigated and resolved.
Funding This work was supported by the National Institutes of Health (NIH RO1 AG 17479, NIH 1F32HL137285-0) and the American Heart Association (AHA14SFRN20600009-02).

Competing interests None declared.

Patient consent for publication Not required.

Provenance and peer review Not commissioned; externally peer reviewed.

\section{REFERENCES}

1 Poole DC, Hirai DM, Copp SW, et al. Muscle oxygen transport and utilization in heart failure: implications for exercise (in)tolerance. Am J Physiol Heart Circ Physiol 2012;302:H1050-H1063.

2 Schalcher C, Rickli H, Brehm M, et al. Prolonged oxygen uptake kinetics during lowintensity exercise are related to poor prognosis in patients with mild-to-moderate congestive heart failure. Chest 2003:124:580-6.

3 Brunner-La Rocca HP, Weilenmann D, Follath F, et al. Oxygen uptake kinetics during low level exercise in patients with heart failure: relation to neurohormones, peak oxygen consumption, and clinical findings. Heart 1999;81:121-7.

4 Chatterjee NA, Murphy RM, Malhotra R, et al. Prolonged mean V02 response time in systolic heart failure: an indicator of impaired right ventricular-pulmonary vascular function. Circ Heart Fail 2013;6:499-507.

5 Kemps HM, Schep G, Zonderland ML, et al. Are oxygen uptake kinetics in chronic heart failure limited by oxygen delivery or oxygen utilization? Int I Cardiol 2010;142:138-44.

6 Smith AA, Cowburn PJ, Parker ME, et al. Impaired pulmonary diffusion during exercise in patients with chronic heart failure. Circulation 1999;100:1406-10.

7 Dhakal BP, Malhotra R, Murphy RM, et al. Mechanisms of exercise intolerance in heart failure with preserved ejection fraction: the role of abnormal peripheral oxygen extraction. Circ Heart Fail 2015;8:286-94.

8 Kitzman DW, Nicklas B, Kraus WE, et al. Skeletal muscle abnormalities and exercise intolerance in older patients with heart failure and preserved ejection fraction. $\mathrm{Am}\rfloor$ Physiol Heart Circ Physiol 2014:306:H1364-H1370.

9 Shimiaie J, Sherez J, Aviram G, et al. Determinants of Effort Intolerance in Patients With Heart Failure: Combined Echocardiography and Cardiopulmonary Stress Protocol. JACC Heart Fail 2015;3:803-14.

10 Haykowsky MJ, Brubaker PH, John JM, et al. Determinants of exercise intolerance in elderly heart failure patients with preserved ejection fraction. J Am Coll Cardiol 2011:58:265-74

11 Abudiab MM, Redfield MM, Melenovsky V, et al. Cardiac output response to exercise in relation to metabolic demand in heart failure with preserved ejection fraction. Eur $J$ Heart Fail 2013;15:776-85.

12 Pandey A, Khera R, Park B, et al. Relative Impairments in Hemodynamic Exercise Reserve Parameters in Heart Failure With Preserved Ejection Fraction: A Study-Level Pooled Analysis. JACC Heart Fail 2018;6:117-26.

13 Borlaug BA, Olson TP, Lam CS, et al. Global cardiovascular reserve dysfunction in heart failure with preserved ejection fraction. J Am Coll Cardio/ 2010;56:845-54 
14 Obokata M, Olson TP, Reddy YNV, et al. Haemodynamics, dyspnoea, and pulmonary reserve in heart failure with preserved ejection fraction. Eur Heart $J$ 2018;39:2810-21.

15 Obokata M, Reddy YNV, Pislaru SV, et al. Evidence Supporting the Existence of a Distinct Obese Phenotype of Heart Failure With Preserved Ejection Fraction. Circulation 2017;136:6-19.

16 Santos M, Opotowsky AR, Shah AM, et al. Central cardiac limit to aerobic capacity in patients with exertional pulmonary venous hypertension: implications for heart failure with preserved ejection fraction. Circ Heart Fail 2015;8:278-85.

17 Henein M, Mörner S, Lindmark K, et al. Impaired left ventricular systolic function reserve limits cardiac output and exercise capacity in HFpEF patients due to systemic hypertension. Int J Cardiol 2013;168:1088-93.

18 Bhella PS, Prasad A, Heinicke K, et al. Abnormal haemodynamic response to exercise in heart failure with preserved ejection fraction. Eur J Heart Fail 2011;13:1296-304.

19 Kitzman DW, Higginbotham MB, Cobb FR, et al. Exercise intolerance in patients with heart failure and preserved left ventricular systolic function: failure of the FrankStarling mechanism. J Am Coll Cardiol 1991;17:1065-72.

20 Massie BM, Conway M, Yonge $\mathrm{R}$, et al. 31P nuclear magnetic resonance evidence of abnormal skeletal muscle metabolism in patients with congestive heart failure. Am J Cardiol 1987;60:309-15

21 Koike A, Hiroe M, Adachi $\mathrm{H}$, et al. Oxygen uptake kinetics are determined by cardiac function at onset of exercise rather than peak exercise in patients with prior myocardial infarction. Circulation 1994;90:2324-32.

22 Sietsema KE, Ben-Dov I, Zhang YY, et al. Dynamics of oxygen uptake for submaximal exercise and recovery in patients with chronic heart failure. Chest 1994;105:1693-700.
23 Koike A, Yajima T, Adachi H, et al. Evaluation of exercise capacity using submaximal exercise at a constant work rate in patients with cardiovascular disease. Circulation 1995;91:1719-24.

24 Weiss K, Schär M, Panjrath GS, et al. Fatigability, Exercise Intolerance, and Abnormal Skeletal Muscle Energetics in Heart Failure. Circ Heart Fail 2017;10.

25 Brunner-La Rocca HP, Weilenmann D, Schalcher C, et al. Prognostic significance of oxygen uptake kinetics during low level exercise in patients with heart failure. Am J Cardiol 1999;84:741-4.

26 Meyer K, Schwaibold M, Hajric R, et al. Delayed V02 kinetics during ramp exercise: a criterion for cardiopulmonary exercise capacity in chronic heart failure. Med Sci Sports Exerc 1998;30:643-8.

27 Katz SD, Maskin C, Jondeau G, et al. Near-maximal fractional oxygen extraction by active skeletal muscle in patients with chronic heart failure. J App/ Physiol 2000;88:2138-42.

28 Maeder MT, Thompson BR, Htun N, et al. Hemodynamic determinants of the abnorma cardiopulmonary exercise response in heart failure with preserved left ventricular ejection fraction. J Card Fail 2012;18:702-10.

29 Phan TT, Abozguia K, Nallur Shivu G, et al. Heart failure with preserved ejection fraction is characterized by dynamic impairment of active relaxation and contraction of the left ventricle on exercise and associated with myocardial energy deficiency. $J$ Am Coll Cardiol 2009;54:402-9.

30 Molina AJ, Bharadwaj MS, Van Horn C, et al. Skeletal Muscle Mitochondrial Content, Oxidative Capacity, and Mfn2 Expression Are Reduced in Older Patients With Heart Failure and Preserved Ejection Fraction and Are Related to Exercise Intolerance. JACC Heart Fail 2016;4:636-45. 